\title{
Ajustes fonético-fonológicos en niños de primer año de educación básica provenientes del sistema educativo público y privado. Incidencia del sexo y el tipo de establecimiento educacional al que asisten
}

\author{
Enzo Fabián Alarcón Acuña ${ }^{1}$ \\ Hernán Mauricio León Valdés \\ Jaime Patricio Soto-Barba \\ Universidad de Concepción \\ Facultad de Humanidades y Artes, Chile \\ Katia Lorena Sáez Carrillo \\ Universidad de Concepción \\ Facultad de Ciencias Físicas y Matemáticas, Chile
}

\section{Resumen}

Antecedentes y objetivo. El estudio de las influencias del nivel sociocultural y el sexo sobre el desempeño fonético-fonológico

\footnotetext{
1 Para correspondencia, dirigirse a: Enzo Fabián Alarcón Acuña (enzoalarconacuna@ gmail.com), ORCID 0000-0002-1418-0052, Hernán Mauricio León Valdés (hleon@udec.cl), ORCID 0000-0002-7986-7509, Jaime Patricio Soto-Barba (jstot@udec.cl ), ORCID 00000001-6513-4534, Departamento de Español, Facultad de Humanidades y Artes; Katia Lorena Sáez Carrillo (ksaez@udec.cl), ORCID 0000-0002-8580-8038, Departamento de Estadística, Facultad de Ciencias Físicas y Matemáticas, Universidad de Concepción, Concepción, Chile. Código postal: 4030000 .
} 
infantil se ha centrado principalmente en los años preescolares $\mathrm{y}$ ha mostrado menos atención sobre niños escolares. Asimismo, escasos estudios analizan cómo estos dos factores interactúan en el desempeño infantil de niños y niñas chilenos y qué tipo de influencias manifiestan en el desempeño fonético-fonológico. El objetivo del estudio es determinar el desempeño fonético-fonológico entre niños y niñas chilenos con desarrollo típico del lenguaje provenientes de un establecimiento privado y de otro municipal. Metodología. Participaron en este estudio 57 participantes, de los cuales 33 sujetos asisten al establecimiento privado (18 niños y 15 niñas) y 24 sujetos asisten al establecimiento municipal (11 niños y 13 niñas). Se aplicó una prueba de repetición de palabras y se analizaron las producciones utilizando una herramienta denominada Pauta de Clasificación de Ajustes Fonético-Fonológicos (CLAFF). Resultados. Los niños del establecimiento municipal obtuvieron un desempeño significativamente menor en los participantes, aunque tales influencias socioculturales no afectan a los niños y las niñas por igual. Para el sexo no hay diferencias significativas entre niños y niñas. Conclusiones. Las influencias del sistema educativo municipal desfavorecen significativamente a los niños y las niñas en los AFF de sílaba y significativamente a las niñas en los AFF de rasgos. No se manifiestan diferencias estadísticas entre niños y niñas de un mismo sistema educativo.

Palabras clave: sistema educativo, nivel sociocultural, sexo, desempeño fonético-fonológico, CLAFF.

\section{PHONETIC-PHONOLOGICAL ADJUSTMENTS IN FIRST-YEAR CHILDREN OF BASIC EDUCATION FROM THE PUBLIC AND PRIVATE EDUCATION SYSTEM. INCIDENCE OF SEX AND THE TYPE OF EDUCATIONAL ESTABLISHMENT THAT THEY ATTEND}

\section{Abstract}

Background and objective. The study of the influences of the sociocultural level and sex on children's phonetic-phonological performance has mainly focused on the preschool years and shown less attention on school. Furthermore, few studies analyze how these two factors interact in the child performance of the Chilean children's and what type of influences they manifest in the phoneticphonological performance. The objective of the study is to determine the phonetic-phonological performance among Chilean boys and girls with typical language development coming from a private and from another public school. Methodology. 57 informants participated in this study, of which 33 subjects attend the private school (18 boys 
and 15 girls) and 24 subjects attend the public school (11 boys and 13 girls). A word repetition test was applied and the productions were analyzed using a tool called Classification of Phonetic-Phonological Adjustments (CLAFF in spanish). Results. The children of the public school obtained a significantly lower performance in the participants, although such socio-cultural influences do not affect boys and girls equally. For the sex, there are no significant differences between boys and girls. Conclusions. The influences of the public educational system significantly disadvantage boys and girls in syllable AFFs and significantly girls in articulatory features AFFs. There are no statistical differences between boys and girls of the same educational system.

Keywords: educational system, sociocultural level, sex, phoneticphonological performance, CLAFF.

Recibido: $09 / 01 / 20 \quad$ Aceptado: $28 / 12 / 20$

\section{FINANCIAMIENTO2}

El presente estudio fue financiado por el proyecto de investigación asociativo VRID UDEC N $\mathrm{N}^{\circ}$ 218.083.036-1.0 y, en lo que respecta a la labor del autor principal, se cuenta con el patrocinio de una beca de estudios CONICYT Programa Formación de Capital Humano Avanzado Beca de Magíster Nacional /2018-221805612.

\section{INTRODUCCIÓN}

Diversos son los factores que ejercen su influencia en la producción del desempeño fonético-fonológico de niños y niñas con desarrollo típico,

2 Adscripción al proyecto de investigación asociativo VRID UDEC N²18.083.036-1.0. Patrocinio de Beca de estudios CONICYT, Programa de Capital Humano Avanzado Beca de Magíster Nacional/2018-221805612. 
tanto en el dominio de la producción de los rasgos articulatorios de los segmentos como el logro de una adecuada estructuración silábica. Entre ellos podemos mencionar, por una parte, el nivel sociocultural como una variable reconocida como un importante determinante en este aspecto, tal como lo señalan estudios realizados en niños y niñas chilenos y en niños y niñas hablantes del francés, español, inglés y portugués. Por otra parte, están las influencias del sexo en el desempeño fonético-fonológico, las que no han sido suficientemente consensuadas en la literatura; en efecto, las investigaciones que han incluido esta variable muestran distintos resultados tanto para la lengua española, como para el inglés, portugués y francés. En cuanto al sexo, los estudios sugieren que constituye una variable que no puede ser obviada en este ámbito. Además, es reconocido que, si se quiere estudiar las diferencias en la capacidad fonético-fonológica entre niños y niñas, los aspectos socioculturales deben incluirse en la investigación.

En los estudios que relacionan el nivel sociocultural y el lenguaje infantil, las investigaciones reportadas utilizan diferentes indicadores para describir hechos que definen este nivel. Entre ellos podemos encontrar la profesión u oficio de los padres, el ingreso económico familiar y el nivel educativo, entre otros. En relación con esto, Moreno (2009) señala que la profesión u oficio de los padres es considerada como una variable sociocultural que permite la estratificación o determinación de estatus en las poblaciones estudiadas. En Chile, la estratificación de establecimientos de educación privada y pública está fuertemente asociada a factores socioculturales y económicos. En el primer tipo de establecimiento, se concentran familias de altos ingresos provenientes de nivel sociocultural medio-alto, mientras que, en el segundo establecimiento, se encuentran familias de bajos ingresos provenientes de nivel sociocultural medio-bajo. Esto ha permitido que el tipo de sistema educativo sea utilizado en Chile para estratificar a la población infantil en estudios lingüísticos (Balladares, Marshall \& Griffiths 2016). Junto a lo anterior, los establecimientos chilenos de educación pública utilizan un indicador denominado IVE-SINAE (Índice de Vulnerabilidad Escolar del Sistema Nacional de Asignación con Equidad). Este permite valorar la condición de vulnerabilidad escolar asociada a factores de riesgo tales como factores económicos, educativos, culturales y sociales (SINAE 2005).

La revisión de las distintas investigaciones indica que el desempeño fonético-fonológico de niños y niñas aparece relacionado con el nivel sociocultural en los estudios del habla infantil en diversas lenguas, donde se presentarán los hallazgos más relevantes para lenguas como el portugués, el inglés y el francés; para luego referirse a distintos dialectos del español. Para el caso del portugués, se han encontrado diferencias significativas entre sujetos de escuelas privadas y públicas a partir de los 4 años de edad, 
el que mediante una prueba de denominación evaluaron el inventario fonológico y los procesos fonológicos (Silva et al. 2012). Para la misma lengua, Ceron, Gubiani, Oliveira \& Keske-Soares (2017) utilizan una prueba de denominación y una herramienta para determinar el porcentaje de consonantes correctas, con lo que confirman la diferencia entre los sistemas educativos municipales y privados, reforzando la importancia del nivel sociocultural al estudiar la adquisición fonética-fonológica en una muestra de 733 sujetos de 3 a 8;11 años de edad. En la lengua inglesa, los informes son sorprendentemente contradictorios, así; Dodd, Holm, Zhua \& Crosbie (2003), en un estudio normativo efectuado en 684 niños y niñas, al aplicar una prueba de denominación y de repetición silábica no encuentran diferencias significativas en el desarrollo fonológico de sujetos preescolares y escolares provenientes de estratos alto y bajo, mientras que Roy et al. (2014) sí encuentran diferencias significativas a favor del estrato medio-alto en comparación con el estrato medio-bajo, en el rendimiento fonético-fonológico en tareas de repetición de palabras en 376 sujetos preescolares de 3;6 a 4;11 años. En la lengua francesa, Barbu et al. (2015) utilizaron pruebas de denominación para evaluar el rendimiento fonológico mediante la observación del fenómeno de la "liaison" en 262 niños y niñas, encontrando diferencias significativas a favor de los niños y niñas de nivel alto, comparados con niños y niñas de nivel bajo en los rangos etarios de $2 ; 6$ a $6 ; 4$ años.

En otros dialectos del español, se reportan rendimientos semejantes para 601 sujetos preescolares peruanos de 3 a 5 años provenientes de establecimientos municipales y privados en la producción fonológica de fonemas vocálicos y consonánticos, lo que fue evaluado en la producción de narraciones (Dioses et al. 2006). En el dialecto del español peninsular, Monfort y Juárez (1988), evaluaron 516 sujetos con tareas de denominación, lo que les permitió reportar un mejor rendimiento fonético-fonológico en aquellos sujetos preescolares del grupo de clase alta en relación con los sujetos preescolares de clase baja, lo cual se acentúa en los rangos de edad de 3 a 3;6 años para luego asemejarse entre los 5;6 y 6;6 años de edad, aunque tales diferencias no alcanzan a ser significativas. En cambio, en otro estudio realizado en el español peninsular (González, 1989), aplica una prueba de repetición de palabras a 198 sujetos, en los que se encuentran diferencias significativas en la producción de fonemas y procesos de simplificación fonológica (PSF) a favor de los sujetos de nivel sociocultural medio y alto provenientes de escuelas privadas, en comparación con sujetos de nivel bajo provenientes de escuelas públicas en el rango etario de 3 a 6;11 años.

En cuanto al español de Chile, en el estudio realizado por Herrera y Pandolfi (1990) se evalúan 20 niños con una edad promedio de 3 años, 
pertenecientes a niveles socioculturales alto y bajo, en los que describen las manifestaciones fonéticas y fonológicas, sin declarar su metodología para obtener los datos, informando una leve superioridad del estrato sociocultural alto. Otro estudio informa diferencias estadísticas significativas a favor del nivel sociocultural medio alto al evaluar la producción de PSF en 180 niños y niñas preescolares de 3 a 6 años utilizando tareas de imitación diferida (Coloma, Pavez, Maggiolo y Peñaloza 2010). Con metodología similar, Pavez et al. (2009) encuentran tendencias semejantes en 360 preescolares y escolares de ambos sexos. Por otra parte, en un estudio fonético-fonológico en varones chilenos de 7 a 9 años de edad, se aplica una prueba de denominación y se reportan diferencias por nivel sociocultural en la producción de fonemas, grupos consonánticos y diptongos, resultando un mejor rendimiento en el estrato sociocultural medio que en el bajo (Vivar y León 2007).

Antes de abordar los estudios realizados sobre la relación entre el habla infantil y el sexo, resulta de gran interés citar un estudio de Karmiloff y Karmiloff-Smith (2005), quienes afirman que el cerebro de las niñas se desarrolla más tempranamente que el de los niños, permitiéndoles controlar un poco antes su aparato articulador. Esto sugiere que las niñas pudieran presentar un mejor desempeño fonético-fonológico al inicio de la etapa escolar, en la que esta habilidad se encuentra en el proceso de culminación de su desarrollo en el rango etario de niños de primero básico (Aguilar 2005; Bosch 2004; González 1989; Owens 2003; Susaníbar, Huamaní y Dioses 2013; Vivar y León 2009).

A continuación, se presentarán diferentes reportes que consideran los hallazgos más relevantes para lenguas como el portugués, el inglés y el francés, para luego dar cuenta de los estudios realizados en el español. En estudios para el portugués de Brasil, Ferrante, Van Borsel y Britto Pereira $(2008,2009)$ no reportan diferencias por sexo en el análisis del inventario fonológico, porcentaje de consonantes correctas y manejo de PSF al evaluar mediante tareas de denominación a 240 sujetos de 3 a 8 años de nivel sociocultural medio-alto provenientes de escuelas privadas; fenómeno que se repite al comparar con pruebas de denominación la adquisición fonético-fonológica de niños y niñas preescolares y escolares de escuelas públicas y privadas (Ceron et al. 2017; Silva et al. 2012). Para la lengua inglesa, los resultados son heterogéneos; por un lado, Dodd et al. (2003) no reportan diferencias estadísticamente significativas en el rendimiento fonético-fonológico al aplicar tareas de denominación y repetición silábica en su muestra de niñas y niños preescolares; fenómeno que se repite en el estudio de Roy et al. (2014) cuando aplican tareas de repetición de palabras a 376 niños y niñas preescolares. Por otro lado, se advierten diferencias estadísticamente significativas por sexo en el rango de edad de 5;6 a 6;11 
años, mostrando las niñas un mejor rendimiento en la producción de grupos consonánticos y fonemas $/ \mathrm{h} / \mathrm{y} / \mathrm{d} /$ (Dodd et al. 2003). En niños franceses, se han encontrado diferencias significativas por sexo en el nivel sociocultural bajo al observar el fenómeno de la "liaison" utilizando tareas de denominación en 262 sujetos en los rangos etarios de 2;6 a 6;4 años, donde las niñas muestran un desempeño significativamente superior al de los niños; sin embargo, tales diferencias no se advierten entre niños y niñas de nivel sociocultural alto (Barbu et al. 2015).

En cuanto a la lengua española, en niños y niñas preescolares peruanos de 3 a 5 años no se informan diferencias significativas en la producción fonológica de fonemas vocálicos y consonánticos en tareas de producción narrativa (Dioses et al. 2006). En otro dialecto del español, una investigación en niñas y niños dominicanos de 2 a 6;5 años observaron la adquisición de fonemas consonánticos donde ambos grupos alcanzan a la misma edad un nivel de dominio fonético-fonológico similar (De la Fuente 1986, citado en Rodríguez y Berruecos 1993, p. 46). Asimismo, para el español peninsular, se han reportado diferencias no significativas entre niñas y niños de 3 a 6 años en la producción de fonemas y PSF, tanto en tareas de repetición (González 1989) como en tareas de denominación (Monfort y Juárez 1988) y, también en tareas de producción espontánea respecto a la producción de metátesis (Diez-Itza y Martínez 2003). En el español de Chile, los estudios fonéticofonológicos no reportan diferencias significativas en el uso de PSF entre niñas y niños preescolares (Coloma et al. 2010) y niñas y niños preescolares y escolares (Pavez et al. 2009).

En este ámbito de estudio, en Chile, como parte de una línea de investigación en fonética y fonología aplicadas a la fonoaudiología se ha utilizado la Pauta de Clasificación de Ajustes Fonético Fonológicos (CLAFF). Esta pauta no se adscribe a una corriente teórica específica, sino que pretende entregar al investigador información que podría interpretarse desde diferentes corrientes teóricas. Desde una perspectiva fonética-articulatoria, la pauta CLAFF permite describir y organizar las modificaciones efectuadas por un niño cuando intenta reproducir el habla adulta. A dichas modificaciones se les ha denominado ajustes fonético-fonológicos (AFF), es decir, realizaciones del habla infantil que no coinciden con el modelo fonológico del español estándar (Soto-Barba, León y Torres 2011). Esta pauta puede aplicarse a corpus obtenidos a través de diversos recursos metodológicos como el análisis de habla espontánea, semi espontánea y no espontánea mediante tareas de repetición, tareas de denominación, tareas de recontado, entre otras. CLAFF permite distinguir, a modo general, ajustes a los rasgos articulatorios y ajustes a la sílaba, lo que se explica de forma más detallada en la metodología de la presente investigación. Utilizando la pauta CLAFF, 
se ha descrito la producción fonético-fonológica infantil empleando, inicialmente, la metodología de recontado de cuentos para obtener el corpus para realizar el análisis en niños típicos preescolares (Lara, Soto-Barba y León 2011; León 2012; León, Soto-Barba y Ñanculeo 2019), en niños típicos preescolares chilenos escolarizados en inglés (Fuica y Soto-Barba 2014) y niños preescolares con trastornos específicos del lenguaje (TEL) (Torres y Soto-Barba 2016). Recientemente, para facilitar la obtención del corpus, se utilizó la repetición directa de palabras mediante la aplicación de la prueba Test de Repetición Fonético Fonológica (TREFF) en niños típicos preescolares (Hamdan 2017) y, en niños preescolares y escolares con trastornos del espectro autista (Torres, León y Figueroa 2018). En resumen, indistintamente de la metodología utilizada en los informantes preescolares con desarrollo típico, el análisis CLAFF permitió observar que niños de nivel sociocultural alto con desarrollo típico presentaron un mejor desempeño fonético fonológico que niños de nivel sociocultural medio bajo.

Como se constata en la revisión expuesta, los estudios en Chile se han dirigido exclusivamente a preescolares, por lo que existe la necesidad de conocer el desempeño fonético y fonológico de niñas y niños chilenos que asisten a primero básico de establecimientos públicos y privados, toda vez que no se dispone de esta información en una edad en que se inicia el aprendizaje formal de la lectoescritura en el cual contribuyen dichas habilidades lingüísticas (González y Romero 1999).

Considerando la revisión expuesta, el objetivo de la presente investigación es determinar las influencias del tipo de establecimiento y el sexo en el desempeño fonético-fonológico de niñas y niños de primer año de enseñanza básica cuya lengua materna es el español de Chile.

\section{METODOLOGÍA}

\subsection{DISEÑO}

Este estudio es una investigación de tipo no experimental, correlacional, de corte transversal y con un enfoque metodológico cuantitativo. Se consideraron como variables independientes el tipo de establecimiento educacional y el sexo de los escolares. Se trata de variables categóricas, puesto que se establecen dos tipos de sistema educativo, el privado y el municipal y, para el sexo, las categorías de mujeres (niñas) y hombres (niños). 
Como variables dependientes actúan la cantidad y las categorías de ajustes fonético-fonológicos producidos por los participantes, las que se comportan como variables continuas y numéricas.

\subsection{CARACTERIZACIÓN Y SELECCIÓN DE LOS PARTICIPANTES}

El presente estudio considera participantes hispanohablantes de Chile de ambos sexos con desarrollo típico del lenguaje oral e indemnidad anatómica de los órganos fonoarticulatorios que asisten a primer año de enseñanza básica municipal o pública y privada. Para determinar si los participantes presentaban un desarrollo típico del lenguaje se procedió a revisar las fichas escolares de los niños y las niñas, descartándose aquellos que presentaban diagnóstico vigente de alguna alteración comunicativa o presentaban alguna necesidad educativa especial. La indemnidad de los órganos fonoarticulatorios fue establecida mediante la aplicación de una evaluación anatómica y funcional realizada por un fonoaudiólogo para excluir a aquellos sujetos portadores de condiciones que pudieran perturbar la producción del habla de los informantes.

La población estudiada pertenecía a establecimientos de una comuna de la ciudad de Concepción, capital de la región del Biobío en Chile. Para acceder a los participantes, se realizó la solicitud correspondiente a las autoridades de cada sistema educativo y establecimiento, luego se obtuvo la autorización de los padres de los participantes mediante la firma de un consentimiento informado. Además, esta investigación contó con la autorización del Comité de Ética, Bioética y Bioseguridad de la Vicerrectoría de la Institución responsable. Todos aquellos sujetos que cumplieron con los criterios de inclusión fueron seleccionados, los que constituyeron una muestra conformada por 18 niños y 15 niñas provenientes del establecimiento privado, y 11 niños y 13 niñas provenientes del establecimiento municipal; de esta manera, la totalidad de la muestra quedó conformada por 57 sujetos con un promedio de edad de 6;6 años y un rango etario de 5;11 años a 7 años. Como se puede ver en la Tabla 1, los comportamientos de los estadísticos de tendencia central y de dispersión para la edad de los participantes son bastante homogéneos y similares, lo que revela que esta variable se comporta de manera estable. Los sistemas educativos fueron asociados con un nivel sociocultural específico, es decir, el establecimiento privado, al nivel sociocultural medio-alto (NMA) y establecimiento municipal, al nivel sociocultural medio-bajo (NMB), considerando lo señalado por diversos autores (Balladares et al. 2016; Ceron et al. 2017; Dioses et al. 2006; González 1989; Monfort y Juárez 1988; Silva et al. 2012; Vivar y León 
2007). El índice de vulnerabilidad escolar que presenta el establecimiento de educación pública es de un promedio general de $97,44 \%$, informado por las autoridades de la institución.

\begin{tabular}{lcccc}
\hline Edad & Media & D. T & Mediana & Q1-Q3 \\
\hline Total & 6.59 & 0.38 & 6.57 & $6.37-6.79$ \\
$\begin{array}{l}\text { Establecimiento } \\
\text { privado }\end{array}$ & 6.56 & 0.3 & 6.6 & $6.38-6.77$ \\
$\begin{array}{l}\text { Establecimiento } \\
\text { municipal }\end{array}$ & 6.62 & 0.47 & 6.56 & $6.36-6.79$ \\
\hline
\end{tabular}

DT= Desviación estándar, Q1 - Q3= Percentil 25 y 75 respectivamente

Tabla 1. Medidas de tendencia central y de dispersión para la edad de la muestra

\subsection{INSTRUMENTO DE OBTENCIÓN DEL CORPUS}

El corpus utilizado en este estudio se obtuvo mediante la aplicación de un instrumento de repetición de palabras, denominado Test de Repetición Fonético-Fonológica (TREFF). Este test fue diseñado para obtener un corpus homogéneo en los participantes a partir de la repetición de un listado de 104 palabras que elicitan la producción de los fonos del español en todas las posiciones posibles en la sílaba y la palabra; previo a esta aplicación dicho test fue validado por el juicio de dos fonólogos expertos en el habla infantil (Hamdan 2017). En aplicaciones posteriores ha demostrado ser eficaz en evidenciar manifestaciones fonético-fonológicas propias de poblaciones infantiles patológicas (Torres, León y Figueroa 2018). Posteriormente, como parte del proyecto de investigación al cual se adscribe este artículo, fue sometido a un proceso de revalidación y análisis de fiabilidad que demostró que esta prueba cumple con las exigencias psicométricas de validez y fiabilidad (Vásquez 2020).

\subsection{PROCEDIMIENTOS Y MÉTODOS DE ANÁLISIS}

Una vez efectuados los procedimientos para la selección de los participantes, se procedió a aplicar la prueba TREFF en una sala con condiciones físicas y acústicas adecuadas para la tarea por realizar. Cada sesión por participante tuvo un tiempo de duración que no superó los doce minutos y fue grabada en audio digital mediante la utilización de una grabadora Tascam DR-40 a una 
frecuencia de muestreo de $44.1 \mathrm{kHz}$. Posteriormente, se procedió a editar los audios grabados utilizando el programa PRAAT (Boersma \& Weenink 2012), en el que se ejecutaron dos scripts que permitieron segmentar, etiquetar y extraer las 104 palabras producidas por cada informante, las que luego fueron analizadas auditivamente para establecer cuáles eran portadoras de ajustes. Así se obtuvo el corpus del estudio, el que fue analizado fonéticamente y transcrito en una hoja de registro individual por cada informante y utilizando la pauta CLAFF para identificar y clasificar las categorías y tipos de AFF encontrados. En la transcripción fonética realizada, actuaron en primera instancia dos jueces y, en los casos de discrepancia, se utilizó un tercer juez para dirimir el desacuerdo. Todos los jueces poseen formación como fonetistas. Como elemento de apoyo adicional, se utilizó la observación de la imagen espectrográfica de la producción fonética mediante el programa PRAAT.

La pauta CLAFF aplicada en este estudio utiliza los criterios de clasificación que se detallan a continuación. La pauta CLAFF agrupa los AFF en dos grandes categorías, en primera instancia, los AFF realizados sobre los rasgos articulatorios de modo, sonoridad y zona de articulación de los segmentos sonoros producidos. Estos pueden modificarse de manera aislada o combinada, por ejemplo, AFF de modo, AFF de modo y zona con cercanía, AFF de modo y sonoridad, etc. En segunda instancia, se agrupan los AFF efectuados sobre la estructura de la sílaba consistentes en adiciones u omisiones de segmentos y sílabas, los que pueden actuar al inicio, interior o final de la palabra. También se registran los ajustes que consisten en desplazamientos de elementos dentro de la palabra, metátesis, y modificaciones que afecten al núcleo silábico como monoptongación y diptongación (Ver Anexo 1). Es importante señalar que las variaciones de carácter dialectal encontradas en la producción fonético-fonológica de los participantes no son consideradas como AFF, puesto que están presentes en el modelo fonético-fonológico del habla adulta como parte del entorno comunicativo de los niños durante su proceso de adquisición fonológica asociado a diversas influencias como el nivel sociocultural, la zona geográfica, etc. Por lo anterior, no se registran aquellas manifestaciones dialectales descritas para el español chileno (Cerda, Fuentes, Soto-Barba y Hamdan 2015; Figueroa, Soto-Barba y Ñanculeo 2010; Pereira y Soto-Barba 2017; Retamal y Soto-Barba 2017; Soto-Barba, Díaz y Pereira 2015). Es importante precisar que, en este artículo, solo se presentan los resultados por categoría de ajustes que resultan suficientes para explicar los hallazgos más importantes en función del objetivo del estudio, omitiendo la presentación de los tipos de ajustes realizados. 


\subsection{ANÁLISIS DE LOS DATOS}

Los datos obtenidos de cada uno de los participantes fueron tabulados y cuantificados en una base de datos Excel, la cual fue analizada utilizando el software SPSS 24.0. Posteriormente, se realizó un primer análisis exploratorio y descriptivo mediado por el porcentaje y frecuencia de las ocurrencias de ajustes para las variables estudiadas. Luego, se aplicó la prueba de Shapiro Willk, la que determinó que los datos de las variables en estudio para una muestra no aleatoria, no presentaron distribución normal, por lo que no se cumplen los requisitos de aplicabilidad de pruebas paramétricas. Por lo anterior, se aplicó una prueba de comparación por grupos independientes con la prueba no paramétrica $U$ de Mann Whitney, para posteriormente aplicar una ANOVA de Kruskal-Wallis con contrastes post hoc, debido a que el análisis fue estadísticamente significativo en la interacción de las variables estudiadas. Se consideró un nivel de significancia $\alpha=0,05$. Al exponer los resultados de los contrastes, por comodidad se utilizaron las letras a, b y c, para indicar de mejor manera las diferencias al comparar más de dos grupos, de modo que cuando dos grupos poseen una letra en común no presentan diferencias significativas a un valor $\mathrm{p}>0,05$.

\section{RESULTADOS}

Primeramente, se realizó un análisis inicial de tipo comparativo-descriptivo en función de la frecuencia y porcentaje del total de AFF y agrupados de acuerdo con su división en AFF de rasgos y AFF de sílaba, los cuales son comparados considerando las variables en estudio (Ver Tabla 2). Se observa un total de $990 \mathrm{AFF}$ en la muestra, en donde existe un predominio de AFF de rasgos por sobre los AFF de sílaba, independientemente de la variable estudiada. Ambos AFF se expresan de forma constante y homogénea advirtiendo para los ajustes de rasgos un rango que varía entre un $61 \%$ - $63 \%$ $y$, para los ajustes de sílaba un rango que varía entre 37\% - 39\%.

\begin{tabular}{lllll}
\hline Grupo & $\mathrm{n}$ & Total de AFF & Ajustes de Rasgos & Ajustes de Sílaba \\
\hline Total muestra & 57 & 990 & $614(62 \%)$ & $376(38 \%)$ \\
$\begin{array}{l}\text { Establecimiento } \\
\text { privado }\end{array}$ & 33 & 418 & $264(63 \%)$ & $154(37 \%)$
\end{tabular}




\begin{tabular}{lcccc}
$\begin{array}{l}\text { Establecimiento } \\
\text { municipal }\end{array}$ & 24 & 572 & $350(61 \%)$ & $222(39 \%)$ \\
Niños & 29 & 541 & $338(63 \%)$ & $203(37 \%)$ \\
Niñas & 28 & 449 & $276(61 \%)$ & $173(39 \%)$ \\
\hline
\end{tabular}

$\mathrm{n}=$ número de participantes

Tabla 2. Distribución de frecuencias y porcentajes de Ajustes fonético-fonológico por cada grupo

En cuanto al análisis estadístico, en la Tabla 3 se informan las medidas descriptivas de los AFF por categoría de rasgos y sílabas para la totalidad de la muestra, donde los estadísticos de tendencia central se manifestaron superiores en los ajustes de rasgos $(M=10,77$ y $M d n=7)$, comparados con los ajustes de sílaba $(M=6,60$ y $M d n=5)$. De igual manera, hay un mayor rango de varianza en los estadísticos de dispersión para los ajustes de rasgos $(D T=8,89$ y $R I Q=10)$, comparados con los ajustes de sílaba $(D T=6,13$ y $R I Q=6)$, en los cuales estos últimos presentan una menor variabilidad.

\begin{tabular}{llllll}
\hline Variable & Media & D.T. & Mediana & Q1 & Q3 \\
\hline Rasgos & 10,77 & 8,89 & 7 & 5 & 15 \\
Sílaba & 6,60 & 6,13 & 5 & 3 & 9 \\
\hline
\end{tabular}

DT= Desviación estándar, Q1 - Q3= Percentil 25 y 75 respectivamente

Tabla 3. Medidas descriptivas de AFF de rasgos y sílaba en el total de la muestra

La Figura 1 muestra la representación gráfica de la distancia intercuartil de los ajustes de rasgos y sílabas para la totalidad de la muestra. En ella se observa una mayor homogeneidad de los datos en la categoría de AFF de sílaba. También, se aprecia que hay dos participantes con valores atípicos para los AFF de rasgos y tres participantes con valores atípicos para los AFF de sílaba. 
Categorías de AFF del total de la muestra

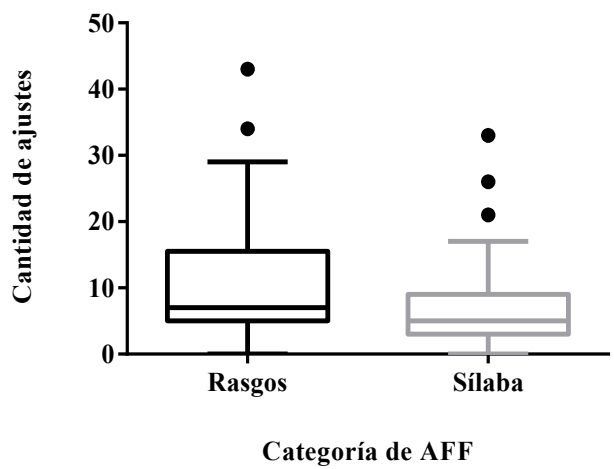

Figura 1. El gráfico de caja representa la variabilidad de los datos del total de la muestra para los AFF de rasgos y AFF de sílaba.

AFF: Ajuste fonético-fonológico

En cuanto a los resultados de los ajustes considerando las variables del estudio, se observó lo siguiente. Primeramente, para comparar los valores de la muestra por grupos emparejados, se aplicó la prueba no paramétrica Test U de Mann Whitney, con el fin de determinar, por una parte, la significación de las diferencias según el tipo de establecimiento (privado y municipal) y, por otra, la significación entre el sexo (ver Tabla 4).

\begin{tabular}{|c|c|c|c|c|c|c|c|c|}
\hline \multirow[b]{2}{*}{ Variable } & & & \multicolumn{2}{|c|}{ Total } & \multicolumn{2}{|c|}{ Rasgos } & \multicolumn{2}{|c|}{ Sílaba } \\
\hline & & $\mathrm{n}$ & $\begin{array}{c}\text { Media } \\
\text { (D.T) }\end{array}$ & $\begin{array}{l}\text { Mediana } \\
\text { (Q1-Q3) }\end{array}$ & $\begin{array}{l}\text { Media } \\
(\mathrm{D} . \mathrm{T})\end{array}$ & $\begin{array}{l}\text { Mediana } \\
\text { (Q1-Q3) }\end{array}$ & $\begin{array}{l}\text { Media } \\
\text { (D.T) }\end{array}$ & $\begin{array}{l}\text { Mediana } \\
\text { (Q1-Q3) }\end{array}$ \\
\hline \multicolumn{9}{|l|}{ Sexo } \\
\hline & Niñas & 28 & $\begin{array}{c}16,04 \\
(13,36)\end{array}$ & $\begin{array}{c}13 \\
(8-17)\end{array}$ & $\begin{array}{c}9,86 \\
(8,19)\end{array}$ & $\begin{array}{c}7 \\
(5-12)\end{array}$ & $\begin{array}{c}6,18 \\
(6,16)\end{array}$ & $\begin{array}{c}5 \\
(4-7)\end{array}$ \\
\hline & & & 18,66 & 16 & 11,66 & 8 & 7,0 & 5 \\
\hline & Niños & 29 & $(14,72)$ & $(8-25)$ & $(9,58)$ & $(5-16)$ & $(6,18)$ & $(3-9)$ \\
\hline \multirow{2}{*}{\multicolumn{2}{|c|}{$\begin{array}{c}\text { Estadígrafo } \mathrm{W} \\
\text { valor } p\end{array}$}} & & \multicolumn{2}{|c|}{757,5} & \multicolumn{2}{|c|}{779} & \multicolumn{2}{|c|}{780} \\
\hline & & & \multicolumn{2}{|c|}{0,3839} & \multicolumn{2}{|c|}{0,5974} & \multicolumn{2}{|c|}{0,6078} \\
\hline
\end{tabular}

\section{Tipo de establecimiento}

Establecimiento

Privado

$12,67 \quad 1$

$33(10,39)$
$(5-17)$

7

$\begin{array}{ccc}8,0 & 7 & 4,67 \\ (6,35) & (4-12) & (4,83)\end{array}$




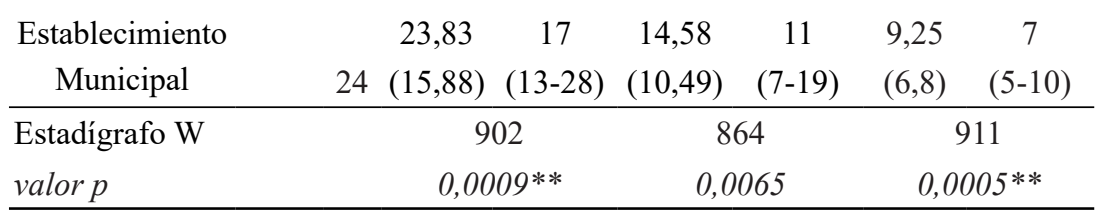

D.T= Desviación estándar. Q1 - Q3= Percentil 25 y 75 .

Test U de Mann Whitney. *Valores significativos $(\mathrm{p}<0.05)$.

Tabla 4. Medidas de tendencia central y de dispersión para los AFF, considerando variables individuales de sexo y tipo de establecimiento

En la Tabla 4 se observa que, para las variables estudiadas, los estadísticos de tendencia central y de dispersión se manifestaron superiores para los ajustes de rasgos, comparados con los ajustes de sílabas. Se alcanzan valores estadísticamente significativos para la variable tipo de establecimiento donde el establecimiento privado se diferencia del establecimiento municipal en los ajustes de sílaba ( $M d n$ establecimiento privado $=4$ y $M d n$ establecimiento municipal $=7 ; W=911 ; p<0,05)$. No obstante, para la variable sexo el análisis estadístico por muestras independiente no arrojó valores significativos para los ajustes los ajustes de rasgos $(W=779 ; p>0,05)$ y para los ajustes de sílaba $(W=789 ; p>0,05)$.

Seguidamente, el comportamiento estadístico de las variables independientes, posterior a la aplicación de la prueba $U$ de Mann Whitney, exige el análisis no paramétrico mediante una ANOVA de Kruskal-Wallis de comparaciones múltiples para evaluar la interacción entre el tipo de establecimiento y el sexo, vinculando las variables y estableciendo más de dos muestras independientes. Esta interacción arrojó diferencias estadísticamente significativas en los ajustes de rasgos $(H=8,28 ; p=0,0398)$ y en los ajustes de sílaba $(H=13,15 ; p=0,0041)$. La prueba de Nemenyi se empleó como procedimiento post hoc, el que viene incorporado a la tabla mediante letras (ver Tabla 5).

\begin{tabular}{|c|c|c|c|c|c|c|c|c|c|}
\hline \multirow[b]{2}{*}{$\begin{array}{c}\text { Tipo de } \\
\text { establecimiento }\end{array}$} & \multirow[b]{2}{*}{ Sexo } & \multicolumn{2}{|c|}{ Total } & & \multicolumn{2}{|c|}{ Rasgos } & & \multicolumn{2}{|c|}{ Sílaba } \\
\hline & & $\begin{array}{l}\text { Media } \\
\text { (D.T.) }\end{array}$ & $\begin{array}{l}\text { Mediana } \\
\text { (Q1-Q3) }\end{array}$ & & $\begin{array}{l}\text { Media } \\
\text { (D.T.) }\end{array}$ & $\begin{array}{l}\text { Mediana } \\
\text { (Q1-Q3) }\end{array}$ & & $\begin{array}{l}\text { Media } \\
\text { (D.T.) }\end{array}$ & $\begin{array}{l}\text { Mediana } \\
\text { (Q1-Q3) }\end{array}$ \\
\hline & Niñas & $\begin{array}{c}9,67 \\
(5,12)\end{array}$ & $\begin{array}{c}11 \\
(5-14)\end{array}$ & b & $\begin{array}{c}6,13 \\
(3,60)\end{array}$ & $\begin{array}{c}6 \\
(4-8)\end{array}$ & & $\begin{array}{c}3,53 \\
(2,62)\end{array}$ & $\begin{array}{c}4 \\
(1-5)\end{array}$ \\
\hline $\begin{array}{c}\text { Establecimiento } \\
\text { Privado }\end{array}$ & Niños & $\begin{array}{c}15,17 \\
(12,93)\end{array}$ & $\begin{array}{c}12.5 \\
(4-22)\end{array}$ & ab & $\begin{array}{c}9,56 \\
(7,72)\end{array}$ & $\begin{array}{c}7 \\
(3-16)\end{array}$ & b & $\begin{array}{c}5,61 \\
(6,01)\end{array}$ & $\begin{array}{c}4.5 \\
(2-8)\end{array}$ \\
\hline
\end{tabular}




\begin{tabular}{|c|c|c|c|c|c|c|c|c|}
\hline & Niñas & $\begin{array}{cc} & 23,38 \\
\text { ab } & (16,18)\end{array}$ & $\begin{array}{c}15 \\
(13-28)\end{array}$ & $\begin{array}{ll} & 14,15 \\
\text { a } \quad(9,92)\end{array}$ & $\begin{array}{c}9 \\
(6-22)\end{array}$ & a & $\begin{array}{c}9,23 \\
(7,65)\end{array}$ & $\begin{array}{c}7 \\
(5-8)\end{array}$ \\
\hline $\begin{array}{c}\text { Establecimiento } \\
\text { Municipal }\end{array}$ & Niños & $\begin{array}{cc} & 24,36 \\
\text { a } & (16,28)\end{array}$ & $\begin{array}{c}18 \\
(15-36)\end{array}$ & $\begin{array}{cc} & 15,09 \\
\text { a } & (11,61)\end{array}$ & $\begin{array}{c}14 \\
(7-19)\end{array}$ & $\mathbf{a}$ & $\begin{array}{c}9,27 \\
(6,02)\end{array}$ & $\begin{array}{c}9 \\
(4-12)\end{array}$ \\
\hline \multicolumn{2}{|c|}{ Estadígrafo $\mathrm{H}$} & \multicolumn{2}{|c|}{12,79} & \multicolumn{2}{|c|}{8,28} & \multicolumn{3}{|c|}{13,15} \\
\hline \multicolumn{2}{|l|}{ valor $p$} & \multicolumn{2}{|c|}{$0.0051 * *$} & \multicolumn{2}{|c|}{$0.0398 *$} & \multicolumn{3}{|c|}{$0.0041 * *$} \\
\hline
\end{tabular}

Test de Kruskal-Wallis. D.T= Desviación estándar. Q1 - Q3= Percentil 25 y 75. Prueba de Nemenyi: Grupos con una letra común no son significativamente diferentes $(p>0,05)$.

Tabla 5. Medidas de tendencia central y de dispersión para el total de AFF, considerando el tipo de establecimiento y el sexo

De la tabla 5 se desprende que, por una parte, para los ajustes de rasgos el análisis post hoc mostró diferencias significativas para el tipo de establecimiento, es decir, entre las niñas del establecimiento privado y las niñas del establecimiento municipal $(M d n$ niñas establecimiento privado $=$ 6 y $M d n$ niñas establecimiento municipal $=9 ; p<0,05)$. Sin embargo, las diferencias por tipo de establecimiento no alcanzaron a ser significativas entre los grupos de niños $(p>0,05)$. Para la variable sexo no hubo diferencias significativas al interior de los establecimientos, esto es, entre niñas y niños del establecimiento privado $(M d n$ niñas establecimiento $=6$ y $M d n$ niños establecimiento privado $=7 ; p>0,05)$ y entre niñas y niños del establecimiento municipal ( $M d n$ niñas establecimiento municipal $=9$ y $M d n$ niños establecimiento municipal $=14 ; p>0,05)$.

En la Figura 2, se observa un diagrama de caja que ilustra las diferencias significativas y la variabilidad de los datos de las cuatro muestras cuando se comparan los ajustes efectuados a los rasgos articulatorios del sonido.

Por otra parte, para los ajustes de sílaba el análisis post hoc reveló diferencias significativas para el tipo de establecimiento en ambos sexos, esto es, entre niñas del establecimiento privado y niñas del establecimiento municipal $(M d n$ niñas establecimiento privado $=4$ y $M d n$ niñas establecimiento municipal $=7 ; p<0,05)$ y entre niños del establecimiento privado y niños del establecimiento municipal ( $M d n$ niños establecimiento privado $=4,5$ y $M d n$ niños establecimiento municipal $=9 ; p<0,05)$. Para la variable sexo no hubo diferencias significativas al interior de los establecimientos, esto es, entre niñas y niños del establecimiento privado $(M d n$ niñas establecimiento privado $=4 \mathrm{y} M d n$ niños establecimiento privado $=4,5 ; p>0,05)$ y entre niñas y niños del establecimiento municipal $(M d n$ 
niñas establecimiento municipal $=7$ y $M d n$ niños establecimiento municipal $=9 ; p>0,05)$.

En la Figura 3, se observa un diagrama de caja que ilustra las diferencias significativas y la variabilidad de los datos de las cuatro muestras.

AFF de rasgos por sexo y tipo de establecimiento

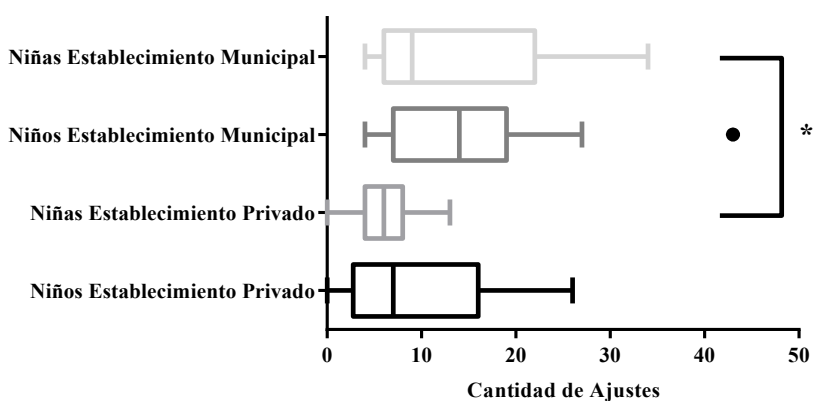

Figura 2. El gráfico de caja representa la variabilidad de los datos de las muestras para los AFF de rasgos según sexo y tipo de establecimiento.

$$
\text { *: } \mathrm{p}<0,05
$$

AFF: Ajuste fonético-fonológico

\section{DISCUSIÓN}

Los resultados del análisis inicial mostraron una marcada preferencia de las niñas y los niños de los dos tipos de establecimientos a efectuar AFF de rasgos por sobre los AFF de sílaba. De modo evidente, lo primero que resalta de estos resultados consiste en que, independientemente del criterio de observación ocupado, en todas las variables analizadas, los ajustes relacionados con los rasgos y sílabas se manifiestan de forma constante y homogénea. Además, se observa un predominio de ajustes efectuados a los rasgos por sobre los ajustes efectuados a la estructura silábica, lo que coincide con otros estudios efectuados con CLAFF en poblaciones con desarrollo típico del habla (León 2012; León et al. 2019). Por un lado, en el estudio de León $(2012,2019)$ los niños de 3 a 4 años presentan dicha tendencia, aunque no se muestra en los niños de 4 a 5 años; sin embargo, tales diferencias son mínimas. Por otro lado, en el estudio de Fuica y SotoBarba (2014) en la muestra de niños de 4 a 5 años escolarizados en inglés, 
también se marca la predominancia para los ajustes de rasgos por sobre los de sílaba. Lo anterior da cuenta de que el dominio fonológico de la sílaba ocurre más tempranamente que el dominio de los rasgos articulatorios de los fonemas, tal como lo reporta el estudio de Ciccia-Gabillo et al. (2009) quienes advierten que el periodo sensible para la adquisición de la sílaba se da entre los 2.6 y 3.6 años, por lo que es esperable que la sílaba tenga mayor estabilidad y dominio fonológico en los participantes de esta investigación. Sin embargo, este antecedente no pretende señalar que todos los tipos de estructura silábica se encuentren adquiridos posterior a esta edad, ya que los participantes del presente estudio aún evidencian estructuras silábicas más complejas que deben ser consolidadas.

Al comparar los resultados considerando los análisis de AFF de rasgos y AFF de sílaba, asociado a las influencias del tipo de establecimiento en el desempeño fonético-fonológico, en esta investigación se encuentran diferencias significativas entre sistemas educativos para la categoría de AFF de sílaba, en los cuales las niñas y los niños del establecimiento municipal muestran un rendimiento fonético-fonológico más descendido, traducido en una mayor manifestación de AFF, diferenciándose de sus pares escolarizados en el establecimiento privado en los que se observa un mejor rendimiento. Estos resultados concuerdan con las investigaciones que advierten las influencias del nivel sociocultural en el desempeño fonético-fonológico de sujetos chilenos (Coloma et al. 2010; Herrera y Pandolfi 1990; Pavez et al. 2009; Vivar y León 2007). En otros dialectos del español, se han reportado las mismas tendencias por cuanto los sujetos pertenecientes al nivel sociocultural bajo presentan un menor desempeño fonético-fonológico que los sujetos de nivel sociocultural alto (Dioses et al. 2006; González 1989; Monfort y Juárez 1988). Sin embargo, en la lengua inglesa, Dodd et al. (2003) no encuentran diferencias asociadas con el nivel sociocultural en sujetos preescolares y escolares; aunque en un estudio más reciente de habla inglesa se revelan diferencias significativas en informantes preescolares, mostrando los sujetos de nivel sociocultural bajo un rendimiento inferior a los sujetos de nivel sociocultural medio-alto en tareas de repetición (Roy et al. 2014). En sujetos hablantes del portugués de Brasil, se ha encontrado la misma ventaja en el nivel sociocultural alto en el uso de PSF, pero solo a partir de los 4 años (Silva et al. 2012), aunque estos resultados no coinciden con estudios más recientes realizados por Ceron et al. (2017) quienes reportan que no se encuentran diferencias significativas entre establecimientos públicos y privados. Sin embargo, ambos estudios difieren en el tamaño y características sociodemográficas de la muestra, así como en su metodología; pues el planteamiento estadístico de Ceron et al. (2017) considera en el análisis la totalidad de la muestra y no emparejados 
por edad y tipo de establecimiento, a diferencia del estudio de Silva et al. (2012) en donde las interpretaciones del análisis estadístico consideran ambas variables mencionadas. En niños franceses, igualmente se advierten las diferencias asociadas con el nivel sociocultural, las cuales indican que los niños y las niñas de nivel sociocultural alto presentan un mejor dominio fonológico del fenómeno de liaison que sus pares de nivel sociocultural bajo en el rango etario de 2;6 a 6;4 años. Además, los autores comentan que las influencias del nivel sociocultural bajo afectan a ambos sexos, aunque en mayor medida a los varones (Barbu et al. 2015).

Siguiendo este mismo planteamiento, pero considerando las influencias del sexo de los participantes en el desempeño fonético-fonológico, podemos mencionar que en la presente investigación no se encuentran diferencias relacionadas con esta variable al interior de los establecimientos educativos. Este hallazgo coincide de manera total con las investigaciones que indican que no existen diferencias significativas en el desempeño fonético-fonológico infantil entre niños y niñas hablantes del español (Coloma et al. 2010; De la Fuente 1986, citado en Rodríguez y Berruecos 1993; Díez-Itza y Martínez 2003; Dioses et al. 2006; González 1989; Monfort y Juárez 1988; Pavez et al. 2009), y entre niños y niñas hablantes del portugués (Ceron et al. 2017; Ferrante et al. 2008, Ferrante et al. 2009; Silva et al. 2012). Continuando con esta variable, en la lengua inglesa también hay coincidencias de acuerdo con los resultados del estudio de Roy et al. (2014) y solo de manera parcial con lo reportado por Dodd et al. (2003) quienes determinan únicamente diferencias por sexo en el rango de edad de 5;6 a 6;11 años. En el francés, la tendencia indicada se ha reportado para todos los niños y niñas de $2 ; 6$ a $6 ; 4$ años de nivel sociocultural alto en el fenómeno de la liaison (Barbu et al. 2015). Pese a las diferencias metodológicas y diferentes lenguas de los estudios mencionados, existe convergencia con los resultados de la presente investigación.

En cuanto a la categoría de AFF de rasgos la presente investigación reveló resultados inesperados; el análisis estadístico efectuado por el ANOVA al interactuar, las variables mostraron que el tipo de establecimiento influencia significativamente al grupo de las niñas, donde el desempeño de las niñas del establecimiento privado fue significativamente mayor al de las niñas del establecimiento municipal; sin embargo, esta variable no afectó de manera significativa a los niños, donde las diferencias no fueron significativas. Dicha tendencia no se manifestó en la categoría de AFF de sílaba, pues el análisis mostró diferencias significativas entre las muestras agrupadas. Esto podría explicarse por la diversidad y variabilidad de ajustes de rasgos que presentan los niños en tareas de repetición, donde se utilizan diferentes estrategias cognitivas que involucran la codificación y decodificación del habla para 
producir de manera correcta una palabra; además en esta investigación se advierte que el dominio de la sílaba presenta un mejor desempeño en comparación con el dominio de los rasgos, por lo que estos resultados apoyan este hallazgo.

Respecto de los resultados obtenidos y la revisión de investigaciones previas que han enfocado sus estudios en torno a las influencias del sexo y el nivel sociocultural en el desempeño fonético-fonológico infantil en diferentes lenguas (Ceron et al. 2017; Coloma et al. 2010; De la Fuente 1986, citado en Rodríguez y Berruecos 1993; Díez-Itza y Martínez 2003; Dioses et al. 2006; Dodd et al. 2003; Ferrante et al. 2008, Ferrante et al. 2009; González 1989; Monfort y Juárez 1988; Pavez et al. 2009; Roy et al. 2014; Silva et al. 2012), se puede advertir que las interpretaciones estadísticas del presente trabajo son diferentes a las de los estudios reportados y escasamente advertidos en la literatura. En efecto, la mayoría de estas investigaciones, efectuadas en lenguas hispanohablantes, inglesa y portuguesa, realizan un tipo de análisis estadístico comparando dos muestras independientes, es decir, por un lado, comparando el tipo de nivel sociocultural y, por otro, comparando el sexo. Sin embargo, en el presente estudio, los resultados derivados del análisis estadístico muestran que las variables independientes tienen un comportamiento diferente y deben ser vinculadas, puesto que los efectos de interacción, influenciados por el nivel sociocultural, no afectan de la misma manera a las niñas y los niños. Así lo demuestra el estudio de Barbu et al. (2015), quienes efectuaron análisis de factores con ANOVA y comparaciones múltiples, revelando que las influencias del nivel sociocultural afectan a ambos sexos en el dominio de la liaison, aunque no de la misma manera, ya que las niñas de nivel sociocultural bajo obtuvieron rendimientos intermedios, es decir, superiores al de los niños del mismo nivel sociocultural e inferiores al de niños y niñas de nivel sociocultural alto, donde en este último grupo los desempeños fueron muy similares. Dicho de otra manera, en este estudio las influencias del nivel sociocultural bajo afectan en mayor medida a los niños. Además, los autores de este estudio refieren que la variable de sexo, de manera independiente, no contribuye a la producción de la liaison y, por ende, debe vincularse con la variable sociocultural.

En síntesis, en esta investigación, se observa el efecto de la interacción entre el sexo y el tipo de establecimiento en el que se desenvuelven los niños y niñas de primero básico, por lo que se debe realizar este tipo de interpretaciones para dirigir el análisis estadístico y analizar las diferencias en el lenguaje infantil cuando se aplican pruebas no paramétricas respecto de muestreos no probabilísticos. Pudiera ser que estas interpretaciones expliquen de mejor manera las diferencias entre los niños y las niñas 
provenientes de establecimientos privados y municipales de este estudio, teniendo en cuenta que el desempeño fonético-fonológico descrito en la presente investigación se comporta de manera similar al reportado en otras investigaciones citadas en este trabajo.

\section{CONCLUSIONES}

Este estudio permitió comprobar que el rendimiento fonético-fonológico de las niñas y los niños del establecimiento privado presentan un mayor desarrollo que el de las niñas y los niños del establecimiento municipal, con lo cual se cumple el objetivo propuesto para este trabajo, aunque de una manera distinta a la supuesta, pues se esperaba encontrar, en el análisis estadístico, resultados significativos comparando únicamente dos muestras independientes, esto es comparando el establecimiento municipal y el establecimiento privado.

Continuando con esta idea, el análisis estadístico del comportamiento de las variables independientes exige la vinculación del sexo y el tipo de establecimiento, lo que conduce a establecer cuatro muestras independientes que deben ser contrastadas. Así, en los resultados, se advierte que las influencias del tipo de establecimiento en el que se desenvuelven los escolares afectan a ambos sexos cuando se consideran los AFF efectuados a la categoría de la sílaba; sin embargo, para los AFF efectuados a los rasgos articulatorios del fonema, las influencias del sistema educativo no afectan a los niños y a las niñas por igual. Dicho de otra manera, las influencias del establecimiento municipal desfavorecen de manera significativa a los niños y las niñas en los AFF de sílaba; no obstante, para los AFF de rasgos, esta variable influencia a un solo sexo de manera significativa, en este caso, a las niñas.

En cuanto a las diferencias por sexo, los resultados de esta investigación cumplen con el objetivo planteado, al igual como se aprecia en los estudios que advierten que no existen diferencias significativas influenciadas por la variable sexo, aunque de una forma distinta a la supuesta, pues de manera independiente se esperaban diferencias significativas en el desempeño fonético-fonológico entre niños y niñas. Sin embargo, los análisis estadísticos e interpretaciones posteriores de esta investigación demuestran que los grupos deben ser comparados teniendo en cuenta el tipo de establecimiento, el que pone de manifiesto la pertenencia a uno u otro nivel sociocultural. De esta manera, no se manifiestan diferencias estadísticas entre niños y 
niñas del establecimiento educativo municipal y entre niños y niñas del establecimiento educativo privado en los AFF de rasgos y en los AFF de sílaba.

\section{ANEXO 1}

Pauta de Clasificación de Ajustes Fonético-Fonológicos

Ajustes fonético-fonológicos efectuados a los rasgos articulatorios de los fonemas

\begin{tabular}{|c|c|c|c|}
\hline & Ajuste de Rasgos & Abreviatura & Ejemplo \\
\hline \multirow{7}{*}{$\begin{array}{c}\text { AFF } \\
\text { aislados }\end{array}$} & Ajuste vocálico & $\overline{\mathrm{V}}$ & $\begin{array}{c}\text { [e.li.'kop.te.ro] por } \\
\text { [e.li.'ko.ti.ro] }\end{array}$ \\
\hline & $\begin{array}{l}\text { Ajuste de vocal } \\
\text { por consonante }\end{array}$ & $\mathrm{VxC}$ & ['kwo.ta] por ['kro.ta] \\
\hline & $\begin{array}{c}\text { Ajuste de } \\
\text { consonante } \\
\text { por vocal }\end{array}$ & $\mathrm{CxV}$ & [dra.' 'ұon] por [dja.' ’on] \\
\hline & $\begin{array}{l}\text { Ajuste de } \\
\text { Sonoridad } \\
\end{array}$ & $S$ & [ра.'reð] por [ра.'ret] \\
\hline & $\begin{array}{l}\text { Ajuste de Zona } \\
\text { con cercanía }\end{array}$ & $\mathrm{ZcC}$ & ['ap.to] por ['al.to] \\
\hline & $\begin{array}{l}\text { Ajuste de Zona } \\
\text { con lejanía }\end{array}$ & $\mathrm{ZcL}$ & ['tren] por ['klen] \\
\hline & $\begin{array}{l}\text { Ajuste de Modo } \\
\text { de articulación }\end{array}$ & M & $\begin{array}{l}\text { [te.'le.fo.no] por } \\
\text { [te.'ne.fo.no] }\end{array}$ \\
\hline
\end{tabular}




\begin{tabular}{|c|c|c|c|}
\hline \multirow{7}{*}{$\begin{array}{c}\text { AFF } \\
\text { combinados }\end{array}$} & $\begin{array}{l}\text { Ajuste de modo y } \\
\text { zona con cercanía }\end{array}$ & $\mathrm{MZcC}$ & [re.'lox] por [re.'lok] \\
\hline & $\begin{array}{c}\text { Ajuste de modo y } \\
\text { zona con lejanía }\end{array}$ & $\mathrm{MZcL}$ & [nan.'du] por [nan.' $\widehat{\mathbf{d} z u}]$ \\
\hline & $\begin{array}{l}\text { Ajuste de modo } \\
\text { y sonoridad }\end{array}$ & MS & ['t $\left.\int \mathrm{i} . \mathrm{ko}\right]$ por ['ni.ko] \\
\hline & $\begin{array}{c}\text { Ajuste de } \\
\text { sonoridad y zona } \\
\text { con cercanía }\end{array}$ & $\mathrm{SZcC}$ & ['ob.x $\left.x^{\mathrm{j} e . t o}\right]$ por ['ot, $\left.\mathrm{x}^{\mathrm{j}} \mathrm{e} . \mathrm{to}_{\pi}\right]$ \\
\hline & $\begin{array}{c}\text { Ajuste de } \\
\text { sonoridad y zona } \\
\text { con lejanía }\end{array}$ & $\mathrm{SZcL}$ & ['af.ta] por ['as.ta] \\
\hline & $\begin{array}{l}\text { Ajuste de modo, } \\
\text { sonoridad y zona } \\
\text { de articulación } \\
\text { con cercanía }\end{array}$ & $\mathrm{MSZcC}$ & [a.ța.'uð] por [a.ta.'uf] \\
\hline & $\begin{array}{l}\text { Ajuste de modo, } \\
\text { sonoridad y zona } \\
\text { de articulación } \\
\text { con lejanía }\end{array}$ & MSZcL & ['sig.no] por ['sis.no] \\
\hline
\end{tabular}

Ajustes fonético-fonológicos efectuados a la estructura de la sílaba

\begin{tabular}{|c|c|c|c|}
\hline & Ajustes de Sílaba & Abreviatura & Ejemplo \\
\hline \multirow{3}{*}{$\begin{array}{c}\text { AFF de } \\
\text { adición de } \\
\text { segmentos }\end{array}$} & $\begin{array}{l}\text { Adición de segmentos } \\
\text { al inicio de palabra }\end{array}$ & Aseg1 & ['d dro.xo] por ['ro.xo] \\
\hline & $\begin{array}{l}\text { Adición de segmento } \\
\text { al interior de palabra }\end{array}$ & Aseg2 & ['ai.yre] por ['ai.re]. \\
\hline & $\begin{array}{l}\text { Adición de segmentos } \\
\text { al final de palabra }\end{array}$ & Aseg3 & ['reik] por ['rei]. \\
\hline \multirow{3}{*}{$\begin{array}{l}\text { AFF de } \\
\text { adición de } \\
\text { sílabas }\end{array}$} & $\begin{array}{c}\text { Adición de sílaba al } \\
\text { inicio de palabra }\end{array}$ & Asill & $\begin{array}{c}\text { [ko.'ko.tau] por } \\
\text { ['kwo.ta]. }\end{array}$ \\
\hline & $\begin{array}{c}\text { Adición de sílaba al } \\
\text { interior de palabra }\end{array}$ & Asil2 & 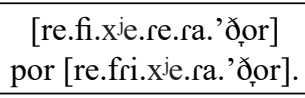 \\
\hline & $\begin{array}{l}\text { Adición de sílaba } \\
\text { al final de palabra }\end{array}$ & Asil3 & $\begin{array}{l}\text { [te.'le.fo.no.lo] } \\
\text { por [te.'le.fo.no] }\end{array}$ \\
\hline
\end{tabular}




\begin{tabular}{|c|c|c|c|}
\hline \multirow{3}{*}{$\begin{array}{c}\text { AFF de } \\
\text { omisión de } \\
\text { segmentos }\end{array}$} & $\begin{array}{c}\text { Omisión de segmento } \\
\text { al inicio de palabra }\end{array}$ & Oseg1 & ['lo. $\beta_{\tau}$ o] por ['glo. $\beta_{T}$ o]. \\
\hline & $\begin{array}{l}\text { Omisión de segmento } \\
\text { al interior de palabra }\end{array}$ & Oseg2 & $\begin{array}{l}\text { [e.li.'ko.te.ro'] por } \\
\text { [e.li.'kop.te.ro']. }\end{array}$ \\
\hline & $\begin{array}{l}\text { Omisión de segmento } \\
\text { al final de palabra }\end{array}$ & Oseg3 & [re.'lo] por [re.'lox] \\
\hline \multirow{3}{*}{$\begin{array}{c}\text { AFF de } \\
\text { omisión de } \\
\text { sílabas }\end{array}$} & $\begin{array}{l}\text { Omisión de sílaba } \\
\text { al inicio de palabra }\end{array}$ & Osill & ['Xlu] por [i.' Ylu]. \\
\hline & $\begin{array}{l}\text { Omisión de sílaba al } \\
\text { interior de palabra }\end{array}$ & Osil2 & 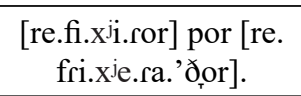 \\
\hline & $\begin{array}{l}\text { Omisión de sílaba } \\
\text { al final de palabra }\end{array}$ & Osil3 & $\begin{array}{l}\text { [te.'le.fo] por } \\
\text { [te.'le.fo.no] }\end{array}$ \\
\hline $\begin{array}{c}\text { Permutación } \\
\text { de sonidos } \\
\end{array}$ & Metátesis & ---------------- & ['ka.ble] por ['kla.be] \\
\hline $\begin{array}{c}\text { Ampliación } \\
\text { de vocal en } \\
\text { diptongo }\end{array}$ & Diptongación & & $\begin{array}{c}\text { [mo.to.'ne.ta] por } \\
{[\text { mo.tjo.'ne.ta] }]}\end{array}$ \\
\hline $\begin{array}{l}\text { Reducción } \\
\text { de diptongo }\end{array}$ & Monoptongación & $\begin{array}{ll}--- \\
-1\end{array}$ & $\begin{array}{c}\text { [au.to.'ma.ti.ko] por } \\
\text { [a.to.'ma.ti.ko] }\end{array}$ \\
\hline
\end{tabular}

\section{REFERENCIAS BIBLIOGRÁFICAS}

Aguilar, Eva. 2005. Comparación entre la adquisición de la fonología castellana y catalana a partir del AREHA y del AREPA. Revista Logopedia Foniatría Audiología 25 (3): 104-14.

Balladares, Jaime; Chlöe Marshall \& Yvonne Griffiths. 2016. Sentence repetition, but not non-word repetition, in Chilean preschoolers. First Language 36 (3): 338-351.

BARBU, ET AL. 2015. Sex differences in language across early childhood: Family socioeconomic status does not impact boys and girls equally. Frontiers in Psychology 6: 1874.

Boersma, Paul \& David Weenink. 2012. Praat: doing phonetics by computer (Computer program). Version 5.3.23. Disponible en http://www.praat.org/.

Bosch, LaURa. 2004. Evaluación fonológica del habla infantil. Barcelona: Masson.

Cerda, Karina; Darío Fuentes; Jaime Soto-Barba y Nahida Hamdan. 2015. Variación diatópica, diastrática y fonética de /s/ codal en el habla de los profesionales chilenos. Onomázein 32 (2): 254-274.

Ceron, Marileda; Marileda Gubiani; Camila de Oliveira \& Márcia Keske-Soares. 2017. Factors influencing consonant acquisition in brazilian portuguese-speaking children. Journal Speech, Language and Hearing Research 60 (4): 759-771.

Ciccia-Gabillo, Cecilia, Oriele Montezuma-del-Castillo, José Elías Ulloa, Gabriela Gabillo-Ciccia, Mery Echenique-Herrera, Gisella Raffo-Catro y Ana María 
Seminario-Olórtigue, A. 2009. La adquisición de la estructura silábica: El caso del castellano limeño. Persona 12: 11-28.

Coloma, Carmen; María Mercedes Pavez; Mariangela Maggiolo y Christian Peñaloza. 2010. Desarrollo fonológico en niños de 3 y 4 años según la fonología natural: Incidencia de la edad y del género. Revista Signos 43 (72): 31-48.

Diez-Itza, Eliseo y Verónica Martínez. 2003. Procesos de metátesis en el desarrollo fonológico de los niños de 3 a 6 años. Psicothema 15 (2): 285-291.

Dioses, Alejandro, Lupe García, María Luisa Matalinares, Abel Cuzcano, Noemí Panca, Janet Quiroz, Carla Fernández, Jenny Castillo. 2006. Análisis psicolingüístico del desarrollo fonético-fonológico de alumnos preescolares de Lima Metropolitana. Revista de Investigación en Psicología 9 (2): 9-32.

Dodd, Barbara; Alison Holm; Hua Zhua \& Sharon Crosbie. 2003. Phonological development: a normative study of British English-speaking children. Clinical Linguistics \& Phonetics 17 (8): 617-643.

Ferrante, Carla; John Van Borsel y Monica Britto Pereira. 2008. Aquisição fonológica de crianças de clase sócio económica alta. Revista CEFAC 10 (4): 452-460.

2009. Análise dos processos fonológicos em crianças com desenvolvimento fonológico normal. Revista da sociedade brasileira de fonoaudiología 14 (1): 36-40.

Figueroa, Mauricio; Jaime Soto-Barba y Marco Ñanculeo. 2010. Los alófonos del grupo consonántico /tr/ en el castellano de Chile. Onomázein 22 (2): 11-42.

Fuica, María Angélica y Jaime Soto-Barba. 2014. Ajustes Fonético-Fonológicos en niños de 4 a 5 años que aprenden inglés como segunda lengua. Literatura y Lingüística 30 (1): 283-299.

GonzÁlez, María José. 1989. Análisis del desarrollo fonológico en sujetos malagueños. Infancia y Aprendizaje 48: 7-24.

González, María José y Juan Francisco Romero. 1999. ¿Influye el desarrollo de la producción fonológica en el aprendizaje de la lectura? Revista de Logopedia, Foniatría y Audiología 19 (2): 61-68.

Hamdan, Nahida. 2017. Desarrollo fonético-fonológico y léxico-semántico de preescolares y su relación con el desempeño lingüístico de sus educadoras de párvulos. Tesis para optar al grado de Magíster en Lingüística Aplicada, Universidad de Concepción.

Herrera, María Oliva y Ana María Pandolfi. 1990. Producción fonológica diastrática de niños menores de tres años. Revista de Lingüística Teórica y Aplicada 28: 101-122.

Karmiloff, Kyra y Anette Karmiloff-Smith. 2005. Hacia el lenguaje. Madrid: Ediciones Morata.

Lara, Isolina; Jaime Soto-Barba y Hernán León. 2011. Ajustes fonético-fonológicos en niños de nivel sociocultural bajo. Ponencia presentada en el XIX Congreso Internacional Sociedad Chilena de Lingüística SOCHIL, Valparaíso, Chile.

LeÓn, Hernán. 2012. Ajustes fonético-fonológicos en niños(as) de habla normal entre 3 a 4 años y 4 a 5 años. Tesis para optar al grado de Doctor en Lingüística, Universidad de Concepción.

León, Hernán; Jaime Soto-Barba y Marco Ñanculeo. 2019. Ajustes fonético-fonológicos en niños de habla típica entre 3 y 4, y entre 4 y 5 años de edad. Onomázein 43: 200-220.

Monfort, Marc y Adoración Juarez. 1988. Registro Fonológico Inducido. Revista de Logopedia, Foniatría y Audiología 8 (2): 88-96.

Moreno, Francisco. 2009. La variación sociolingüística: Las variables sociales. En Principios de sociolingüistica y sociología del lenguaje (4a. ed.). Barcelona: Ariel.

Owens, Robert. (2003). Desarrollo del lenguaje. Madrid: Pearson Educación. 
Pavez, María Mercedes; Mariangela Maggiolo; Christian Peñaloza y Carmen Coloma. 2009. Desarrollo fonológico en niños de 3 a 6 años: incidencia de la edad, el género y el nivel socioeconómico. Revista de Lingüística Teórica y Aplicada 47 (2): 89-109.

Pereira, Daniel y Jaime Soto-Barba. 2017. Bases para la observación del habla subestándar del español chileno: el caso de /d্d/ en el habla de los profesionales chilenos. Ponencia presentada en el XVIII Congreso Internacional de la Asociación de Lingüística y Filología de América Latina (ALFAL), Universidad Nacional de Colombia, Bogotá.

Retamal, Nicolás y Jaime Soto-Barba. 2017. Caracterización fonético-auditiva y fonéticoacústica del fonema vibrante múltiple /r/ en profesionales de 8 ciudades de Chile en situación de lectura en voz alta”. Boletín de Filología 52 (1): 245-271.

Rodríguez, Oralia y María Paz Berrueco. 1993. Trabajos descritos en La adquisición del español como lengua materna: Bibliografia descriptiva, pp. 21-136. México, D.F: Colegio de México. Disponible en http://www.jstor.org/stable/j.ctv47w4c4.

Roy, Penny; Shula Chiat \& Barbara Dodd. 2014. Language and socioeconomic disadvantage: From research to practice. London: City University London.

Silva, Monica; Carla Ferrante; John Van Borsel y Monica Britto Pereira. 2012. Aquisição fonológica do Português Brasileiro em crianças do Rio de Janeiro. Jornal da Sociedade Brasileira de Fonoaudiologia 24 (3): 248-254.

Sistema Nacional de Auxilio Escolar y Becas (SINAE). 2005. Una nueva visión en la construcción de igualdad de oportunidades en la infancia. Disponible en https://www. junaeb.cl/wp-content/uploads/2013/02/libro_junaeb.pdf

Soto-Barba, Jaime; Hernán León y Valeska Torres. 2011. Una propuesta para la clasificación de los ajustes fonético-fonológicos del habla infantil. Onomazein 23 (1): 69-79.

Soto-Barba, Jaime; Erika Díaz y Daniel Pereira. 2015. Variación alofónica y diatópica del fonema /b/ en el español de profesionales chilenos en situación de lectura en voz alta. Literatura y Lingüistica 32 (2): 201-216.

Susaníbar, Franklin; Oscar Huamaní y Alejandro Dioses. 2013. Adquisición fonéticafonológica. EOS 1 (1): 19-36.

Torres, Juan Carlos; Hernán León y Mauricio Figueroa. 2018. Desempeño fonéticofonológico en niños con Trastorno del Espectro Autista (TEA) de pre-kínder a tercero básico. Revista Chilena Fonoaudiología 17: 1-18.

Torres, Valeska y Jaime Soto-Barba. 2016. Ajustes Fonético-Fonológicos en niños con Trastornos Específicos del Lenguaje Mixto (TEL Mixto). Onomázein 33 (1): 69-87

VÁSQuez, SANDra. 2020. Determinación de la validez y fiabilidad del test de repetición fonético-fonológica (TREFF) como instrumento para evaluar el desempeño fonéticofonológico en niños chilenos. Tesis para optar al grado de Magíster en Lingüística Aplicada, Universidad de Concepción.

Vivar, Pilar y Hernán León. 2007. Aplicación del cuestionario para la evaluación de la fonología infantil (C.E.F.I) a una muestra de niños chilenos de diferente nivel socioeconómico. Revista Chilena de Fonoaudiología 8 (1): 17-31.

2009. Desarrollo fonológico-fonético en un grupo de niños entre 3 y 5, 11 años. Revista CEFAC 11 (2): 190-198. 